\title{
A Note on the Exact Green Function for a Quantum System Decorated by Two or More Impurities
}

\section{L. Glasser ${ }^{1,2 *}$}

' Departamento de Física Teórica, Facultad de Ciencias, Universidad de Valladolid, Valladolid, Spain, ${ }^{2}$ Physics Department, Clarkson University, Potsdam, NY, United States

The exact Green function is constructed for a quantum system, with known Green function, which is decorated by two delta function impurities. It is shown that when two such impurities coincide they behave as a single singular potential with combined amplitude. The results are extended to $\mathrm{N}$ impurities and higher dimensions.

Keywords: quantum green function, delta function potential, contact interaction, Schroedinger equation, quantum graph

Pacs: 02.30Gp, 02.30Hq, 03.65Db

\section{INTRODUCTION}

The one dimensional harmonic oscillator or square well, for example, for which the energy -dependent Green function $G_{0}\left(x, x^{\prime} ; E\right)$ is known, have been taken for many years as solvable models for semi-conductor quantum wells [1]. Frequently delta function potentials are placed at various points to simulate defects or impurities. In the case of a single impurity potential, $V(x)=\lambda \delta(x-a)$, the Green function for the composite system is known to be [2]

$$
G\left(x, x^{\prime} ; E\right)=G_{0}\left(x, x^{\prime}\right)+\lambda \frac{G_{0}(x, a ; E) G_{0}\left(a, x^{\prime}\right)}{1-\lambda G_{0}(a, a ; E)}
$$

Universitá degli Studi Guglielmo

Marconi, Italy

Fabiano Andrade

Universidade Estadual de Ponta

Grossa, Brazil

${ }^{*}$ Correspondence:

M. L. Glasser

laryg@tds.net

Specialty section: This article was submitted to

Mathematical Physics,

a section of the journal

Frontiers in Physics

Received: 01 October 2018

Accepted: 11 January 2019

Published: 29 January 2019

Citation:

Glasser ML (2019) A Note on the Exact Green Function for a Quantum System Decorated by Two or More

Impurities. Front. Phys. 7:7.

doi: 10.3389/fphy.2019.00007
In this note a corresponding formula is derived for the case $V(x)=\lambda \delta(x-a)+\mu \delta(x-b)$ On the basis of the analogy of the algebraic structure the result is extended to N-impurities and for a standard interpretation of the Dirac delta function, to higher dimension.

\section{CALCULATION}

We first note that the same argument can be used for the time dependent-, as well as the energy-dependent Green functions, so we shall omit the third argument and write simply $G\left(x, x^{\prime}\right)$.

Beginning with the Dyson equation, noting that $G_{0}(x, y)=G_{0}(y, x)$

$$
G\left(x, x^{\prime}\right)=G_{0}\left(x, x^{\prime}\right)+\int G_{0}(x, y) V(y) G\left(y, x^{\prime}\right) d y,
$$

where the integration extends over the system domain, one has the set of equations

$$
\begin{aligned}
& G\left(x, x^{\prime}\right)=G_{0}\left(x, x^{\prime}\right)+\lambda G_{0}(x, a) G\left(a, x^{\prime}\right)+\mu G_{0}(x, b) G\left(b, x^{\prime}\right) \\
& G\left(a, x^{\prime}\right)=G_{0}\left(a, x^{\prime}\right)+\lambda G_{0}(a, a) G\left(a, x^{\prime}\right)+\mu G_{0}(a, b) G\left(b, x^{\prime}\right), \\
& G\left(b, x^{\prime}\right)=G_{0}\left(b, x^{\prime}\right)+\lambda G_{0}(a, b) G\left(a, x^{\prime}\right)+\mu G_{0}(b, b) G\left(b, x^{\prime}\right) .
\end{aligned}
$$


The linear Equations (4) and (5) are easily solved for $G\left(a, x^{\prime}\right)$ and $G\left(b, x^{\prime}\right)$ :

$$
\begin{aligned}
G\left(a, x^{\prime}\right) & =\frac{G_{0}\left(a, x^{\prime}\right)+\mu\left[G_{0}\left(b, x^{\prime}\right) G_{0}(a, b)-G_{0}\left(a, x^{\prime}\right) G_{0}(b, b)\right]}{D} \\
G\left(b, x^{\prime}\right) & =\frac{G_{0}\left(b, x^{\prime}\right)+\lambda\left[G_{0}\left(a, x^{\prime}\right) G_{0}(a, b)-G_{0}\left(b, x^{\prime}\right) G_{0}(a, a)\right]}{D}
\end{aligned}
$$

with

$$
D=\left[1-\lambda G_{0}(a, a)\right]\left[1-\mu G_{0}(b, b)\right]-\lambda \mu\left[G_{0}(a, b)\right]^{2} .
$$

By inserting (6) and (7) into (3) we obtain the desired expression

$$
\begin{gathered}
G\left(x, x^{\prime}\right)=G_{0}\left(x, x^{\prime}\right) \\
+\frac{1}{D}\left\{\lambda G_{0}(x, a) G_{0}\left(a, x^{\prime}\right)+\mu G_{0}(x, b) G_{0}\left(b, x^{\prime}\right)\right. \\
+\lambda \mu\left[G_{0}(x, a)\left(G_{0}(a, b) G_{0}\left(a, x^{\prime}\right)-G_{0}(b, b) G_{0}\left(b, x^{\prime}\right)\right)\right. \\
\left.\left.+G_{0}(x, b)\left(G_{0}(a, b) G_{0}\left(a, x^{\prime}\right)-G_{0}(a, a) G_{0}\left(a, x^{\prime}\right)\right)\right]\right\} .
\end{gathered}
$$

\section{DISCUSSION}

By setting $\mu$ to 0 (9) reduces to (1), proving this expression as well. The most salient feature of (9) is the denominator $D$ whose zeros form the exact spectrum of the composite system. For example, when $a$ and $b$ coincide, $D$ reduces to $1-(\lambda+$ $\mu) G(a, a)$ and (9) reduces to (1) with $\lambda$ replaced by the amplitude $\lambda+\mu$. I.e., the two impurities combine to form one with combined amplitude. This generalizes the result of Fasssari and Rinaldi [3], for two identical defects symmetrically placed with respect to the center of a harmonic oscillator. An expression similar to (9) has been derived recently by Horing (private communication) for the case of a quantum dot in a magnetic field.

Two further points can be made. Nothing in the derivation of (9) restricts it to the line. If we accept the standard definition $\delta(\vec{x})=\Pi_{j=1}^{d} \delta\left(x_{j}\right)$, then (9), and its consequences, are valid for $d$-dimensional quantum systems. This has been proven functiontheoretically for the three dimensional quantum dot with two symmetrically placed identical impurities by Albeverio et al. [4].

A second observation is that $D$ is simply the Cramer determinant for the pair of simultaneous linear Equations (4) and (5). In the case of impurity potential

$$
V(x)=\sum_{j=1}^{N} \lambda_{j} \delta\left(x-a_{j}\right)
$$

there will be $N$ such equations and the determinant is easily evaluated. The general result is

If a quantum system having Green function $G_{0}(x, y)$ is decorated with $N$ delta function impurities $\lambda_{j} \delta\left(x-a_{j}\right)$, $j=1,2, \cdots N$, then the new energy levels are the roots of

$$
D_{N}=\prod_{j=1}^{N} A_{j j}-\sum_{j=2}^{N}(-1)^{j} \sum_{1 \leq k_{1}<\cdots k_{j} \leq N} A_{k_{1} k_{2}} A_{k_{2} k_{3}} \cdots A_{k_{j} k_{1}}=0
$$

where $A_{l m}=\delta_{l m}-\lambda_{l} G_{0}\left(a_{l}, a_{m}\right)$.

Thus,

$$
\begin{aligned}
D_{3}=\prod_{j=1}^{3} & {\left[1-\lambda_{j} G_{0}\left(a_{j}, a_{j}\right)\right]-\sum_{i<j} \lambda_{i} \lambda_{j} G_{0}\left(a_{i}, a_{j}\right) G_{0}\left(a_{j}, a_{i}\right) } \\
& \quad+\lambda_{1} \lambda_{2} \lambda_{3} G_{0}\left(a_{1}, a_{2}\right) G\left(a_{2}, a_{3}\right) G_{0}\left(a_{3}, a_{1}\right),
\end{aligned}
$$

which reduces to the $N=1$ and $N=2$ cases appropriately and shows that any two coinciding impurities coalesce as indicated above.

Note that if all the $\lambda$ s and $a$ 's coincide then

$D_{N}=\left(1-\lambda G_{0}(a, a)\right)^{N}-\sum_{j=2}^{N}\left(\begin{array}{c}N \\ j\end{array}\right) \lambda^{j} G_{0}(a, a)^{j}=\left(1-N \lambda G_{0}(a, a)\right)$.

Equation (11) might offer a new approach to KronigPenney-type systems for periodic or random unit cells.

Finally, it should be pointed out that the work in this note is paralleled in the theory of quantum graphs introduced by Linus Pauling about 1930 to describe electrons in molecules which has developed into a sophisticated and important branch of quantum physics [5]. For relations of this discipline to the present work see the papers by Andrade et al. [6] and Andrade and Severini [7].

\section{AUTHOR CONTRIBUTIONS}

The author confirms being the sole contributor of this work and has approved it for publication.

\section{ACKNOWLEDGMENTS}

The author thanks Prof. S. Fassari and Prof. L. M. Nieto for helpful comments and acknowledges the financial support of MINECO (Project MTM2014-57129-C2-1-P) and Junta de Castilla y Leon (VA057U16). The author thanks a referee for pointing out the relevance to quantum graphs. 


\section{REFERENCES}

1. Albeverio S, Gesztesy F, Hoegh-Krohn R, Holden H. Solvabe Models in Quantum Mechanics. Providence, RI: AMS-Chelsea (2004).

2. Glasser ML, Nieto LM. The energy level structures of a variety of one-dimensional confining potentials and the effects of a local singular perturbation. Can J Phys. (2015) 93:1-9. doi: 10.1139/cjp-201 5-0356

3. Fassari S, Rinaldi F. On the spectrum of the Schroedinger Hamiltonian of the one-dimensional harmonic oscillator perturbed by two identical attractive point interactions. Rep Math Phys. (2012) 69: 353-70.

4. Albeverio S, Fassari S, Rinaldi F. Spectral properties of a symmetric threedimensional quantum dot with a pair of identical attractive impurities symmetrically situated around the origin. Nanosyst Phys Chem Math. (2016) 7: 268-89. doi: 10.17586/22208054201672268289

5. Berkolaiko G, Kuchement P. Introduction to Quantum Graphs. Providence, RI: AMS Mathematical Surveys and Monographs 186 (2013).
6. Andrade FM, Schmidt GM, Vicentini E, Cheng BK, da Luz MGE. Green's Function approach to quantum graphs: An overview. Phys Rep. (2016) 647:1. doi: 10.1016/j.physrep.2016.07.001

7. Andrade FM, Severini S. Unitary equivalence between the Green's Function and Schroedinger approaches for quantum graphs. arXiv:1808.01154v1.

Conflict of Interest Statement: The author declares that the research was conducted in the absence of any commercial or financial relationships that could be construed as a potential conflict of interest.

Copyright (c) 2019 Glasser. This is an open-access article distributed under the terms of the Creative Commons Attribution License (CC BY). The use, distribution or reproduction in other forums is permitted, provided the original author(s) and the copyright owner(s) are credited and that the original publication in this journal is cited, in accordance with accepted academic practice. No use, distribution or reproduction is permitted which does not comply with these terms. 